\title{
Educación Inclusiva en El Salvador. Una Reflexión desde las Políticas Educativas
}

\section{Inclusive Education in El Salvador. A Reflection from the Educational Policies}

\author{
Cristina Aracely Muñoz Morán* \\ Universidad Evangélica de El Salvador, El Salvador
}

\begin{abstract}
El presente artículo de revisión tiene como objetivo analizar el estado actual de las políticas educativas relacionadas con la inclusión en El Salvador, a partir de diferentes consideraciones como la normativa vigente, el significado de exclusión educativa, la multicausalidad de los problemas educativos, la intersectorialidad, la influencia del contex to o la transformación de las prácticas escolares. Luego, de dicho análisis se presenta una reflexión ético-filosófica sobre la importancia que tiene el reconocimiento de la persona en la política educativa como elemento que promueve la sinergia entre inclusión, equidad y justicia social.
\end{abstract}

Descriptores: Derecho a la educación; Política educativa; Desigualdad social; Participación; El Salvador.

Therefore, this review article aims to analyze the current state of educational policies related to inclusion in El Salvador, based on different considerations such as current legislation, the meaning of educational exclusion, the multiple causes of educational problems, cooperation among sectors, the influence of context or the transformation of school practices. Then, this analysis presents an ethical-philosophical reflection on the importance of the recognition of the person in educational policy as an element that promotes the synergy between inclusion, equity and social justice.

Keywords: Right to education; Educational policy; Social inequality; Participation; El Salvador.

\section{Introducción}

La educación inclusiva implica una transformación radical de los paradigmas educativos, pues propone pasar de un enfoque basado en la homogeneidad a una visión de la educación plural y heterogénea (Blanco, 2006). Además, para incidir en la consolidación de las políticas educativas que promueven la inclusión es necesario tener en cuenta el incremento de las desigualdades sociales, la diversidad cultural y la creciente crisis de cohesión social (López, 2009). Ahora bien, difícilmente se puede separar la inclusión de la realidad sociocultural en que se dinamiza, así como también no se pueden dejar de lado a actores clave como el Estado, la sociedad civil o la familia.

Por tanto, al abordar la inclusión como un problema de políticas educativas es necesario valorar qué propuestas se han elaborado, cómo se están desarrollado, si están funcionando o no (Terigi, 2014). Y esta revisión es la que se realiza en el presente artículo, a fin de reflexionar sobre el proceso que se ha implementado en El Salvador, ya que una transformación gradual hacia una escuela inclusiva, requiere de una revisión y mejora

$\begin{array}{lll}\text { *Contacto: cristina.munoz@uees.edu.sv } & \text { Recibido: } & 30 / 10 / 2018 \\ & 1^{a} \text { Evaluación: } & 15 / 12 / 2018 \\ \text { ISSN: 0718-7378 } & \text { Aceptado: } & 04 / 03 / 2019 \\ \text { www.rinace.net/rlei/ } & & \end{array}$


continua, pues no se puede reducir a una ley o una acción puntual con un alcance temporal limitado (Payá Rico, 2010). Entonces, se hace necesario considerar también "los niveles de inclusión social y los alcances de las brechas de equidad en la adquisición de competencias y aprendizajes" (Opertti, 2009, p. 4). Esto debido a que la inclusión sin su dimensión éticofilosófica de reconocimiento de la persona, se queda como una propuesta sin fundamento, al no ir más allá de la escuela para transformar la sociedad.

\section{Estado del arte sobre la política de educación inclusiva}

Al valorar la educación inclusiva desde un enfoque sistémico se considera que la educación es un sistema que refleja la sociedad global, es decir, representa la concepción de persona que predomina en el contexto en el que se desarrolla el hecho educativo. Por tanto, dicha representación del ser humano también conlleva implícita o explícitamente una forma de entender la vida, las relaciones humanas, el saber o las diferencias individuales. Entonces, si no se visibiliza que históricamente el sistema educativo ha sido excluyente, difícilmente se logrará "organizar una sociedad que sea capaz de dar oportunidades de aprendizaje a todos" (Aguerrondo, 2008, p. 77).

Además, es necesario reflexionar un poco más sobre cómo influye en el proceso de inclusión educativa el momento socio-histórico actual, en el que la solidez de las cosas se interpreta como una amenaza, el conocimiento y los vínculos se espera que sean de uso instantáneo, y donde lo que mejor se vende es la diferencia y no la semejanza (Bauman, 2008). En este escenario se rescata en cierta medida el valor que se le otorga a la diferencia, el cual es un elemento relevante para la educación inclusiva, ya que permite examinar cuál es la concepción de escuela que se tiene y cuál es el propósito de la educación en la sociedad actual (Miles y Singal, 2010).

Por otra parte, como afirma Sánchez de Horcajo (1991), "la educación dentro de una determinada sociedad cambiará con el tiempo y en la medida que cambie la sociedad misma" (p. 126). Es por ello, que la educación ha trascendido su función tradicional como formadora de recursos humanos para optar por la formación de los ciudadanos (López, 2005). Dicho cambio implica pasar del reproductivismo social a poner la mirada en la relación que se establece entre la educación y la sociedad, e incluso si se arriesga un poco más implica afectar los vínculos humanos (Bauman, 2008; López, 2005).

Entonces, en este escenario las políticas educativas tienen un rol fundamental, pues son las que recogen las demandas y las expectativas de la sociedad en lo que se refiere al aporte que se espera de la escuela en la formación de los futuros ciudadanos. Y existen diferentes propuestas para analizar las políticas que se implementan en educación, una de ellas es la elaborada por Terigi (2014), quien propone que es necesario identificar las condiciones sociales y educativas que las políticas atienden o dejan de atender, y valorar cómo funcionan éstas a nivel local.

Además, la propuesta de la autora implica abordar la inclusión como problema de las políticas educativas, razón por la cual se seleccionó para reflexionar sobre el estado actual de dichas políticas en el país. Ahora bien, ya que dicho análisis se realizará desde el contexto salvadoreño es necesario mencionar que la inclusión se entiende en relación a todos los grupos que pueden ser vulnerables de exclusión y especialmente a los estudiantes 
con discapacidad, esto de acuerdo a la tipología propuesta por Ainscow, Booth y Dyson (2006).

El análisis que se presenta a continuación se realizó a partir de ocho consideraciones (Terigi, 2014), las cuales son: derecho a la educación más allá de la dimensión normativa, ampliación del significado de exclusión educativa, multicausalidad de la desescolarización y el rezago escolar, importancia de la multisectorialidad, valoración de los problemas educativos a partir del contexto, intensidad y extensión de las políticas, compromiso del Estado con la consolidación de las políticas educativas y transformación de las prácticas escolares en el sistema educativo regular.

\section{Derecho a la educación más allá de la dimensión normativa}

A nivel regional, América Latina ha tenido diferentes avances en lo relacionado a la legislación como consecuencia de la ratificación de la Convención de los Derechos del Niño (Fondo de las Naciones Unidas para la Infancia, 2006), la Convención sobre los Derechos de las Personas con Discapacidad (ONU, 2007) y otras convenciones internacionales como la de Educación para Todos o los Objetivos de Desarrollo Sostenible (UNESCO, 2017). Sin embargo, todavía existe una desarticulación entre lo que se propone y su puesta en ejecución, por lo que es clave colocar la mirada en las acciones que hagan posible la transformación.

La situación de El Salvador es similar a la planteada anteriormente, pues hay poca coherencia entre las leyes y las políticas que se han diseñado para garantizar el derecho a la educación y su respectiva implementación, pues, aunque se cuenta con un marco normativo, falta impulsar las estructuras que le den seguimiento. Así, el derecho a la educación de todo ciudadano es reconocido tanto en la Constitución de la República de El Salvador (Asamblea Legislativa de El Salvador (1983), como en la Ley General de Educación (Asamblea Legislativa de El Salvador, 1996). Ahora bien, para garantizar dicho derecho y cumplir con los compromisos de la Educación para Todos y los Objetivos de Desarrollo Sostenible, el Ministerio de Educación ha impulsado en las últimas décadas diferentes planes educativos, que a continuación se describen:

- 1995-2005. El Plan Decenal de Educación (Ministerio de Educación, 1995) surge gracias a la reforma educativa que se desarrolló en el país después del conflicto armado. Los ejes de ese plan fueron: cobertura, modernización institucional, mejoramiento de la calidad y formación de valores humanos, éticos y cívicos; los cuales fueron retomados en el año 2000, en los Desafíos de la Educación en el Nuevo Milenio (Ministerio de Educación, 2000).

- 2004-2009. Plan Nacional de Educación 2021 (Ministerio de Educación, 2005), el cual planteó los siguientes objetivos: formación integral de la persona, escolaridad de once grados para toda la población, formación técnica y tecnología del más alto nivel, y desarrollo de la ciencia y la tecnología para el bienestar de la sociedad. Y sus líneas estratégicas fueron: acceso a la educación, efectividad de la educación básica y media, competitividad y buenas prácticas de gestión.

- 2009-2014. Plan Social Educativo: Vamos a la escuela (Ministerio de Educación, 2009), propone rediseñar la escuela con la participación de la familia y la comunidad para mejorar los aprendizajes. Y propone las siguientes líneas estratégicas: acceso y permanencia, currículo pertinente y aprendizaje 
significativo, dignificación y desarrollo profesional docente, fortalecimiento de la gestión institucional, formación permanente de jóvenes y adultos, educación superior e investigación, ciencia y tecnología.

- 2010. Política de Educación Inclusiva (Ministerio de Educación, 2010b) tiene como objetivo trasformar el sistema educativo por medio de la eliminación de las barreras para el aprendizaje y la participación de diferentes actores. Esta política plantea cuatro áreas de acción: normativa y políticas de gestión, prácticas de gestión pedagógica, cultura escolar y comunitaria, ambientes educativos y recursos estratégicos. También se contextualizó el Índice de inclusión (Ainscow y Booth, 2000) a la realidad educativa salvadoreña, y así surgió la Adecuación del índice de inclusión al contexto educativo salvadoreño (Ministerio de Educación, 2010a). Y en él se incorpora la dimensión de territorios inclusivos, es decir, las prácticas educativas que favorecen la participación de los estudiantes y la comunidad en el aprendizaje, tanto dentro como fuera de la escuela.

- 2014-2019. Plan Nacional de Educación en Función de la Nación (Ministerio de Educación, 2015a), el cual se está implementando por medio de diferentes ejes estratégicos: profesionalización docente, primera infancia, evaluación educativa, ambientes escolares, educación equitativa, inclusiva y de calidad; currículo nacional, condiciones para la creación de conocimiento e innovación, educación de adultos, reforma institucional y legislación vigente.

Por otra parte, en la última década también se ha incluido a la sociedad civil en la definición de la educación que el país necesita, un primer paso se dio en el 2004 con la formación de la Comisión Presidencial para el Desarrollo de la Sociedad del Conocimiento (Ministerio de Educación, 2005). Luego, en 2015 se conforma el Consejo Nacional de Educación, el cual es un espacio promovido por el Estado para iniciar una trasformación hacia una educación integral, universal y de calidad. Y para ello elaboraron el Plan El Salvador Educado (Consejo Nacional de Educación, 2016), el cual plantea seis desafíos: escuela libre de violencia, docentes de calidad, atención integral a la primera infancia, doce grados de escolaridad universal, educación superior e infraestructura. A continuación, se presenta un análisis comparativo de las líneas estratégicas o programas de acción que se han propuesto en los planes y las políticas educativas de los últimos veinte años (cuadro 1).

Como se puede observar en el cuadro 1, las áreas que son incluidas por la mayoría de los planes educativos son: educación inclusiva, infraestructura, currículo y gestión. Luego, las áreas que no todos los planes incluyen son las de: atención a la primera infancia, escolaridad universal y desarrollo docente (dignificación y formación). Por otra parte, hay áreas que son priorizadas puntualmente por determinados planes como es el caso del acceso y la permanencia, la formación de adultos o la educación superior.

Ahora bien, llama la atención que la cultura escolar y comunitaria sea incluida únicamente en la Política de Educación Inclusiva (Ministerio de Educación, 2010b) o que sólo en el Plan El Salvador Educado se aborde el fenómeno de la violencia en la escuela (Consejo Nacional de Educación, 2016). En el caso de la violencia, se considera que es un fenómeno tanto estructural como simbólico, pues ya es una forma naturalizada de opresión que la cultura salvadoreña ha internalizado y legitimado como parte de la convivencia cotidiana (PNUD, 2018). Entonces, en este escenario se hace necesario que los esfuerzos que se realizan desde las políticas públicas tengan a la base una visión de inclusión que implique 
a todos los ciudadanos en las aspiraciones comunes, donde todos sean respetados y valorados por ellos mismo (Powell y Tutt, 2002). Y en la medida en que esto se logre se podrán tener las condiciones necesarias para reconstruir el tejido social que se ha debilitado en las últimas décadas, y en ese proceso la educación tiene un papel clave para aportar a la construcción de una verdadera cohesión social.

Cuadro 1. Análisis comparativo de las líneas estratégicas o programas de acción de los planes educativos de las últimas dos décadas

\begin{tabular}{|c|c|c|c|c|c|c|c|}
\hline & 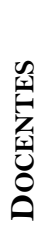 & 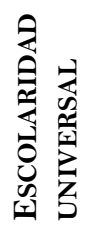 & 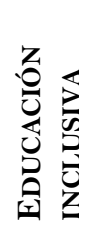 & 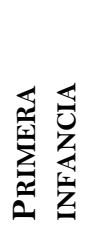 & 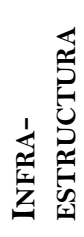 & 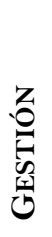 & 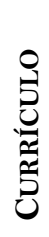 \\
\hline $\begin{array}{l}\text { Plan Decenal de Educación y Desafíos de la } \\
\text { Educación en el Nuevo Milenio }\end{array}$ & $\mathrm{X}$ & - & - & - & - & $\mathrm{X}$ & $\mathrm{X}$ \\
\hline Plan Nacional de Educación 2021 & - & $\mathrm{X}$ & $\mathrm{X}$ & - & $\mathrm{X}$ & $\mathrm{X}$ & $\mathrm{X}$ \\
\hline Plan Social Educativo: Vamos a la escuela & $\mathrm{X}$ & - & $\mathrm{X}$ & $\mathrm{X}$ & $\mathrm{X}$ & $\mathrm{X}$ & $\mathrm{X}$ \\
\hline Política de Educación Inclusiva & - & - & $\mathrm{X}$ & - & $\mathrm{X}$ & $\mathrm{X}$ & $\mathrm{X}$ \\
\hline $\begin{array}{l}\text { Plan Nacional de Educación en Función de la } \\
\text { Nación }\end{array}$ & $\mathrm{X}$ & - & $\mathrm{X}$ & $\mathrm{X}$ & $\mathrm{X}$ & - & $\mathrm{X}$ \\
\hline Plan El Salvador Educado & $\mathrm{X}$ & $\mathrm{X}$ & $\mathrm{X}$ & $\mathrm{X}$ & $\mathrm{X}$ & - & - \\
\hline
\end{tabular}

Fuente: Elaboración propia.

\section{Ampliación del significado de exclusión educativa}

Una manera de comprender la exclusión educativa es a partir de valorar la inclusión como un constructo social, pues es necesario que la persona se sienta parte del colectivo, donde todos se sientan valorados y respetados por sí mismos (Powell y Tutt, 2002). Así, la exclusión educativa puede estar asociada a la escasa importancia que se le brinda al reconocimiento de la diferencia, y esto conlleva a que se pueda excluir "con o sin justificación desde las dinámicas políticas, la sociedad y el mercado, y aún desde los principales ámbitos de socialización (la familia y la escuela)" (Ministerio de Educación, 2010b, p. 16).

Por otra parte, factores como la pobreza, condición de discapacidad, pertenencia a pueblos originarios, enfermedad crónica o trabajo infantil, entre otros, pueden determinar la exclusión desde el sistema educativo, si se configuran como mecanismos de marginación social propios de dicho sistema. Ahora bien, en palabras de Braslavsky (citada en Aguerrondo, 2008) define que existen tres tipos de marginación educativa:

- Marginación por exclusión total, es decir, no se logra el ingreso al sistema educativo. En 2015, el 5.6\% de los niños de 7 años y el 10.3\% de los adolescentes de 15 años estaban fuera del sistema educativo (Consejo Nacional de Educación, 2016). Por tanto, para garantizar el acceso y la permanencia en la escuela hay que incidir en problemas sociales, tales como la violencia, el trabajo infantil, la delincuencia, la pobreza, entre otros (Ministerio de Educación, 2010b).

- Marginación por exclusión temprana, o sea la deserción del sistema educativo formal antes de que las habilidades básicas se hayan consolidado. En el año 2000, el indicador de supervivencia al sexto grado fue de $66.10 \%$ y en el 2012 ascendió al 83.80\%, es decir, aumentó en 18 puntos (Ministerio de Educación, 2015c). Sin 
embargo, solo seis de cada diez estudiantes que ingresan al sistema se mantienen en él hasta noveno grado de educación básica y, solo cinco de diez estudiantes que comienzan en primaria llegan a bachillerato (Consejo Nacional de Educación, 2016).

- Marginación por inclusión, que implica la fragmentación del sistema educativo en segmentos de diferente calidad, lo que en muchas ocasiones conlleva a que no se garantice la adquisición los conocimientos que corresponden al nivel educativo del estudiante. En El Salvador, existe tanto una oferta educativa pública como privada, que abarca desde el nivel de educación inicial hasta la educación superior, aunque está variedad de alternativas predomina en la zona urbana, pues en el área rural la oferta a la que tienen acceso los estudiantes es mayoritariamente pública. También, años atrás el Ministerio de Educación desarrolló un programa de educación acelerada para dar respuesta a los estudiantes en situación de rezago escolar, pues en un año escolar se podían cursar dos años académicos y así reducir gradualmente la brecha de edad con sus compañeros (Ministerio de Educación, 2015c).

Entonces, al comprender la exclusión educativa como un fenómeno generado por factores tanto externos como internos, es necesario que diferentes actores unan esfuerzos para "prevenir y erradicar la exclusión educativa por medio de acciones directas e indirectas que fortalezcan las dinámicas inclusivas en la escuela y las comunidades" (Ministerio de Educación, 2010b, p. 25). Y este ha sido uno de los retos que el Ministerio de Educación ha priorizado desde el 2009 con el Plan Social Educativo (Ministerio de Educación, 2009), pues ha ejecutado diferentes programas de apoyo a la familia y a los estudiantes, tales como: dotación de uniformes y útiles escolares, alimentación, educación básica para la población joven y adulta, recreación, arte y cultura, jóvenes talentos, Escuela Inclusiva de Tiempo Pleno, entre otros (Ministerio de Educación, 2015c).

Finalmente, es necesario reconocer que los programas que el Ministerio de Educación ha implementado para apoyar a las familias no bastan, pues si bien la alimentación o los útiles escolares pueden incidir hasta cierto punto en el rendimiento académico, dichos programas no cuestionan los fundamentos del sistema educativo ni sus planes, por lo que no llegan a proponerse cambios estructurales. Por ello, toma relevancia la valoración de la exclusión como una falta de reconocimiento de la persona, lo que implica "no tener la oportunidad de desarrollar los procesos de pensamiento que permitan entender, convivir y desarrollarse en un mundo complejo" (Aguerrondo, 2008, p. 65). Y desde esta valoración al país le falta camino por recorrer, para pasar de un abordaje asistencialista de la exclusión educativa a una propuesta de transformación radical basada en el reconocimiento de la persona y sus derechos.

\section{Multicausalidad de la desescolarización y el rezago escolar}

La desescolarización y el rezago escolar no pueden comprenderse como fenómenos aislados, ya que pueden producirse por factores individuales, familiares, demográficos, sociales o escolares. Así, en el caso de la deserción escolar influye el acceso temprano al trabajo para contribuir al ingreso familiar, donde para los hombres implica trabajar fuera de la casa y para las mujeres participar en las tareas del hogar (Consejo Nacional de 
Educación, 2016). También se observa una discrepancia al explorar las causas de deserción de acuerdo con la zona geográfica de residencia de los estudiantes, pues quienes residen en el área urbana desertan en su mayoría para trabajar mientras que los estudiantes de la zona rural dejan la escuela por falta de interés, lo cual "podría vincularse a deficiencias en el aprendizaje o a una falta de correspondencia entre la realidad vivida y lo que se estudia” (Consejo Nacional de Educación, 2016, p. 64).

Mientras que la deserción causada por la violencia se da en el plano comunitario, pues "la inseguridad asociada a la presencia de las pandillas recrudece las cifras de deserción y violencia, en particular, en los niveles del Tercer Ciclo de Educación Básica y en Bachillerato" (Consejo Nacional de Educación, 2016, p. 20). También, la violencia se puede dar en el plano familiar, generando desestabilización e incluso desintegración del hogar, lo cual con frecuencia afecta la asistencia del estudiante a la escuela.

Y ante estas realidades el docente no sabe qué hacer, pues en la formación inicial no se les han brindado herramientas y quienes están ya en las escuelas no han desarrollado aún las competencias que necesitan para abordar adecuadamente las situaciones de violencia o vulnerabilidad social que viven los estudiantes, tanto de manera preventiva como paliativa (Consejo Nacional de Educación, 2016). Por tanto, es importante que desde la escuela se creen espacios de reflexión sobre la educación para la no violencia, que permitan establecer relaciones basadas en el diálogo, la participación y el reconocimiento de la persona.

Por otra parte, el rezago escolar puede darse por un ingreso tardío a la escuela o por la repitencia de grados, esto implica que los estudiantes muchas veces no tienen la misma edad que sus compañeros de clase. Estas situaciones se dan con mayor frecuencia en los primeros grados de educación básica, ya sea porque los padres consideran que los niños aún no tienen la edad para asistir a la escuela o por las dificultades que pueden presentar en la adquisición de la lectoescritura (Consejo Nacional de Educación, 2016). Luego, a partir del séptimo grado "las razones se complican y van desde la falta de cobertura territorial, hasta problemas sociales como la pobreza, el embarazo adolescente, la migración y la inseguridad" (Consejo Nacional de Educación, 2016, p. 63).

Luego, al acercarse a los resultados de aprendizaje que alcanzan los estudiantes también se pueden observar desigualdades, las cuales se pueden dar por diferentes factores (Blanco y Duk, 2011):

- Contexto socioeconómico y cultural. El Salvador es considerado como un país de ingresos medios bajos (Organización de las Naciones Unidas para la Educación, 2015), que se encuentra afectado por violencia social, migraciones, desestructuración familiar, trabajo infantil o delincuencia. Por otra parte, en los últimos años se han dado esfuerzos para revitalizar el nahuát como lengua materna (Ministerio de Educación, 2015c).

- Tipo de escuela y entorno de aprendizaje. El clima escolar de las escuelas salvadoreñas muchas veces se ve afectado por la inseguridad y la violencia (Consejo Nacional de Educación, 2016). Y la gestión escolar promueve diversas acciones para lograr aprendizajes significativos con la participación de la comunidad educativa (Ministerio de Educación, 2008a).

- Tiempo efectivo de aprendizaje. En El Salvador se han establecido 200 días lectivos, los estudiantes asisten semanalmente a la escuela entre 18 y 25 horas 
de acuerdo con el nivel educativo en que se encuentren (Ministerio de Educación, 2008b). Ahora bien, es difícil establecer cuán efectivo es el tiempo de aprendizaje.

- Asistencia a educación de la primera infancia. En el país preocupa que "solo se atiende al 3\% de la población que la necesita, que las escuelas parvularias puras están mayormente concentradas en el área urbana" (Ministerio de Educación, 2015a, p.13). Ahora bien, cabe destacar que desde la elaboración de la Política Nacional de Educación y Desarrollo Integral de la Primera Infancia (Ministerio de Educación, 2010c), se propuso un nuevo modelo de atención basado en un enfoque de derechos y desarrollo integral. También se actualizaron los programas de estudio para dicha población y los de la formación de los futuros docentes en educación inicial y parvularia (Ministerio de Educación, 2015c).

- La calidad de los docentes. Generalmente estos carecen de un "marco conceptual y de los instrumentos metodológicos para hacer frente a la diversidad de expectativas y necesidades de los educandos" (Opertti, 2009). En 2012 se crea la Política Nacional de Desarrollo Profesional Docente (Ministerio de Educación, 2012), a fin de fortalecer la formación docente tanto inicial como en servicio. Y en 2015, había 31,085 maestras y 14,786 maestros laborando en el sector público; de los cuales un $81.97 \%$ tienen el nivel académico de profesorado, $7.20 \%$ licenciatura, $6.22 \%$ bachillerato académico y un $4.61 \%$ otros grados (Ministerio de Educación, 2015b).

Además, desde el Plan Social Educativo (Ministerio de Educación, 2009) se definió como una línea estratégica la equidad en el acceso y la permanencia en el sistema educativo, especialmente los estudiantes que se encuentran en situación de vulnerabilidad social. Y para apoyar esto, el Ministerio de Educación brinda los útiles escolares, los uniformes y alimentación a todos los estudiantes de los centros educativos públicos, desde educación inicial hasta educación media.

En el país los datos de deserción escolar en educación primaria han disminuido, en el año 2000 se tenía un porcentaje global de 6.55, mientras que para el 2012 se ha reducido a 3.32 puntos porcentuales. También ha mejorado la tasa neta de escolarización en la enseñanza preescolar, pues pasó del 40\% en 1999 al 62\% en 2012 (UNESCO, 2015). Y en la enseñanza primaria la tasa neta global de escolarización subió en más de 10 puntos, en el año 1999 era de 85\% y en 2012, 95\% (Ministerio de Educación, 2015b; UNESCO, 2015).

Por otra parte, para dar respuesta al rezago escolar en el 2005 se inició el Programa Redes Escolares Efectivas en los 100 municipios más pobres del país con el objetivo de "ampliar las oportunidades para completar la educación básica a niños, niñas y jóvenes de las zonas rurales de extrema y alta pobreza y con mayor rezago educativo" (Ministerio de Educación, 2015b, p. 26). Dicho programa ya no se está implementando, y a partir del 2009 se han desarrollado los programas: Cerrando la brecha del conocimiento y Un niño, una computadora (Ministerio de Educación, 2009). Con dichos programas se busca brindar capacitación y recursos tecnológicos a las escuelas con un alto porcentaje de rezago escolar.

Finalmente, la multicausalidad de la deserción y del rezago escolar no debe conllevar a la simplificación de estos, pues quienes no asisten a la escuela han pasado por muchas situaciones tanto dentro como fuera de la escuela antes de dejar de asistir a ella (Terigi, 2014). Este planteamiento explica parcialmente las limitaciones que ha tenido la política pública en el país, pues, aunque tiene una visión multicausal de la deserción y del rezago 
escolar, en la práctica brinda respuestas superficiales, como la dotación de recursos escolares, programas de alimentación o capacitaciones; no logra articular a los diferentes actores que se necesitan para incidir directamente en las causas que mantienen las barreras para el aprendizaje y la participación.

\section{Importancia de la intersectorialidad}

La consolidación de la educación inclusiva será posible en la medida que también se desarrollen políticas económicas y sociales que den respuesta a los factores que generan exclusión y marginación. Por tanto, uno de los restos de la escuela es ser sensible a las demandas y expectativas del entorno (Krichesky, 2006), por ello es imprescindible vincular el nivel central con el local, tanto para que las políticas educativas se diseñen desde y para el contexto como para generar las condiciones que permitan la autonomía de la escuela en la búsqueda de apoyos a nivel local.

En 2010 con la Política Nacional de Educación y Desarrollo Integral de la Primera Infancia se abre un espacio para que la atención de la niñez sea abordada desde un enfoque intersectorial, pues se crean comités integrados por diferentes instancias (Ministerio de Educación, 2010c). Luego, en 2011 se crea la Mesa Técnica Intersectorial para la Educación y Desarrollo Integral para la implementación del modelo de educación y desarrollo integral de la primera infancia (Ministerio de Educación, 2015c). También participan organizaciones no gubernamentales, municipalidades y cooperantes, aunque a la fecha aún no se cuenta con investigaciones o evaluaciones que muestren cómo han funcionado las mesas intersectoriales, cuáles han sido sus aciertos u obstáculos en su proceso de implementación.

Entonces, la educación inclusiva debería "extenderse a las escalas geográficas de la política local y la política estatal además del espacio escolar" (Rambla et al., 2008, p. 89). En este contexto, en el país falta proponer más iniciativas que impliquen un abordaje intersectorial para la mejora de los aprendizajes, tanto a nivel local como nacional, no basta incluir a los diferentes actores en el diseño de las propuestas, también es necesario que participen en la toma de decisiones sobre su implementación y evaluación.

\section{Valoración de los problemas educativos a partir del contexto}

$\mathrm{Al}$ abordar los problemas educativos del país es importante partir del contexto en el cual se desarrollan, ya que la educación es un tema de interés y relevancia social. Entonces, primero es necesario que los equipos docentes y las familias se pregunten si la propuesta educativa que orienta la práctica pedagógica en la escuela responde al contexto social en el que desarrollan (López, 2007). Ahora bien, desde aquí puede encontrarse un obstáculo, pues, aunque las familias y la comunidad contribuyan en actividades puntuales de la escuela o en la elaboración inicial de los proyectos educativos, generalmente son excluidos de la toma de decisiones sobre los aprendizajes, por lo que la participación política de dichos actores queda limitada (Hurtado y Muñoz, 2017).

Por tanto, si el proyecto educativo no se construye de manera colectiva y tomando en cuenta el contexto socio-histórico, puede caer en un mero cumplimiento burocrático de la norma. En cambio, si la realidad "es mirada desde los sujetos deviene en un conjunto de 
espacios de construcción: es el papel del momento histórico en la medida en que en éste ocurre la intervención de la práctica” (Zemelman, 2005, p. 86), y esta elaboración participativa compromete a cada uno de los actores inmersos en el proceso.

Finalmente, si bien es necesario valorar que el desarrollo de vínculos entre diferentes personas es un proceso complejo, también hay que reconocer que si no se generan los espacios donde se permita el encuentro con el otro, será "poco probable que los miembros de un sector de la sociedad sientan a los de otro como conciudadanos, como parte del mismo grupo social, la misma historia o la misma identidad" (López, 2007, p. 27). Así, el encuentro, la participación y el reconocimiento de la persona son elementos que articulados por la gestión escolar permitirán dinamizar aprendizajes desde y para el contex to donde se desarrollen.

\section{Intensidad y extensión de las políticas}

$\mathrm{Al}$ analizar las políticas educativas en términos de intensidad y extensión, se brinda otra manera de comprenderlas. Así, Terigi (2014) considera que la extensión se refiere al alcance de la política en relación con la población a la que está dirigida, mientras que la intensidad implica "tanto la profundidad de los cambios como la exigencia de instancias de diseño y ajuste en el nivel institucional mismo, con implicación de diversos actores y creación de funciones específicas" (p. 227).

La Política de Educación Inclusiva (Ministerio de Educación, 2010b) ha priorizado la extensión, es decir, abarcar a la niñez y adolescencia que se encuentra en riesgo de exclusión educativa por diversos motivos. Y desde dicha política se han promovido algunas acciones que favorecen una mayor intensidad en los cambios, tales como:

- Estrategia del Docente de Apoyo a la Inclusión, la cual pretende mejorar la atención educativa de los estudiantes en riesgo de exclusión, por medio del apoyo a los distintos actores de la comunidad educativa (Ministerio de Educación, 2017b).

- Centros de Orientación y Recursos, son espacios que brindan apoyo y orientación psicopedagógica a estudiantes, docentes y familias para garantizar el acceso y la permanencia de calidad en el sistema educativo (Ministerio de Educación, 2017a).

- Comités Departamentales de Apoyo a la Inclusión, son estructuras que fueron creadas para dinamizar los procesos de inclusión educativa en el territorio, por medio de la participación de diferentes actores (Ministerio de Educación, 2016b).

Si bien dichas acciones buscan consolidar la escuela inclusiva, aún no son conocidas en todo el territorio, lo que dificulta que se logren articular con los esfuerzos que las escuelas realizan diariamente. Además, a la fecha no existe un plan de seguimiento y evaluación de estas estrategias para valorar el aporte que brindan.

\section{Compromiso del Estado con la consolidación de las políticas educativas}

El avance de una política educativa depende en gran medida del grado de compromiso que el Estado manifieste a través de sus diferentes instancias, ya que solo "es posible conseguir 
progresos cuando los Estados tienen un compromiso con la justicia social que se concreta en el desarrollo de políticas que tienen como foco central la búsqueda de la equidad y la cohesión social” (Blanco y Duk, 2011, p. 49). Además, la educación inclusiva con frecuencia es percibida como un medio para superar la pobreza, pero para ello se necesitan algunas condiciones básicas que contribuyan a que las personas puedan aprovechar las oportunidades de aprendizaje que se le presenten (Opertti, 2009).

Sin embargo, no sólo corresponde al Estado contribuir a generar condiciones de educabilidad (López, 2005), sino también a la sociedad civil, la familia y la escuela, donde cada actor tiene un rol y responsabilidades frente al aprendizaje. Así, las condiciones de educabilidad deben comprenderse como "un concepto relacional, en tanto se define en la tensión entre los recursos que el niño porta y los que la escuela espera o exige de él” (López, 2007, p. 49).

Entonces, en lo que se refiere al compromiso del Estado salvadoreño con la Política de Educación Inclusiva (Ministerio de Educación, 2010b) puede considerarse que es fluctuante, pues no ha sido un eje transversal en las acciones desarrolladas por el sistema educativo en sus diferentes niveles. Por ejemplo, en el organigrama del Ministerio de Educación, el Departamento de Apoyo al Desarrollo la Inclusión Educativa depende de la Gerencia Nacional de Educación de Primera Infancia, desarticulado de los demás niveles educativos (Ministerio de Educación, 2016a). También la educación inclusiva ha quedado en segundo plano en el proceso de profesionalización docente y en la atención a la primera infancia.

Por tanto, es necesario pensar las políticas educativas como movimientos horizontales y no como tradicionalmente se suele hacer de arriba hacia abajo, y este cambio implica una transformación gradual:

Desde las autoridades de un gobierno estatal hasta un maestro en donde la toma de decisiones, despliega transformaciones y más transformaciones en el desarrollo desordenado de la acción política. Donde unas implican y otras transforman a las otras de modo rizomático (Telló, 2016, p. 57)

Y sumado a esta transformación es importante definir los mecanismos que le den seguimiento a la implementación de la política y dentro de los cuales están los procesos evaluación de los resultados que permiten un reajuste y una retroalimentación constante (Pedro y Puig, 1999). En el caso de El Salvador, actualmente no hay una estructura que se encargue específicamente de evaluar y dar seguimiento a las políticas y los planes educativos que se están implementando.

\section{Transformación de las prácticas escolares en el sistema educativo regular}

Difícilmente se puede hablar de inclusión educativa sino se logra el equilibro entre calidad educativa y atención a los diferentes grupos sociales desde sus contextos. Por tanto, para lograr dicho equilibrio es necesario generar acciones de cambio en los siguientes aspectos (Blanco y Duk, 2011):

- Accesibilidad de los programas y servicios educativos a los grupos excluidos. En el país se cuenta con diferentes programas de apoyo, tales como modalidades flexibles para estudiantes que trabajan o que viven en la zona rural, educación 
acelerada, alfabetización de adultos, educación especial para estudiantes con discapacidad intelectual moderada y círculos de familia para la primera infancia, entre otros (Ministerio de Educación, 2015c).

- Establecimiento de objetivos de equidad de manera transversal en todas las políticas sociales. La noción de equidad está presente en las siguientes políticas: Política de Educación Inclusiva (Ministerio de Educación, 2010b), Política de Equidad e Igualdad de Género (Ministerio de Educación, 2016), Política Nacional de Atención Integral a las Personas con Discapacidad (Asamblea Legislativa de El Salvador, 2014), Política Nacional de Educación y Desarrollo de la Primera Infancia (Ministerio de Educación, 2010c) y la Política Nacional de Juventud (Ministerio de Gobernación, 2013).

- Desarrollo de sistemas integrales de protección social que combinen prestaciones básicas universales y apoyos específicos a poblaciones en situación de mayor vulnerabilidad. Entre los programas más significativos están: educación inicial, alimentación y salud escolar, paquetes escolares, un vaso de leche, Cerrando la brecha del conocimiento, un niño una computadora (Ministerio de Educación, 2015c).

- Mayor inversión en la primera infancia. La inversión en educación parvularia pasó de 31 millones de dólares en 2002 a 56 millones en 2010, experimentando una disminución de casi 4 millones al 2011, ya que se asignaron 52.36 millones (Fondo de las Naciones Unidas para la Infancia, 2012).

También es importante reconocer que los estudiantes tienen el derecho de acceder a un currículo diseñado para satisfacer sus necesidades, en un ambiente donde se sientan valorados por sí mismos (Powell y Tutt, 2002). Y en este escenario el rol del docente es clave pues es quien mediará la transformación de la escuela hacia la inclusión y por ello, debe ser "sensible a las diferencias y con competencias para trabajar en contextos complejos, socioculturalmente diversos y con grupos heterogéneos" (Blanco y Duk, 2011, p. 53).

Generalmente, los equipos docentes realizan la planificación y la adecuación curricular de manera colegiada, partiendo del perfil del estudiante y su contexto, pero aún hay escuelas donde esto no se realiza por completo (Hurtado y Muñoz, 2017). Por otra parte, los procesos de formación sobre educación inclusiva han contribuido a la toma de conciencia del derecho a la educación de toda persona, independientemente de sus características, respetando las diferencias de cada uno de los actores educativos y buscando generar relaciones más horizontales entre los mismos (Hurtado y Muñoz, 2017).

\section{Conclusiones}

La educación salvadoreña enfrenta un gran reto, pues como afirma López (2007) "ya no somos todos partes de un mismo grupo, se va diluyendo la existencia" (p. 32), lo que conlleva a que la sociedad esté fragmentada, pues en muchas ocasiones se invisibiliza a las personas o se las trata como que no existieran, ya sea porque no cumplen los cánones del saber dominante o por no aportar al crecimiento económico (Santos, 2010). Entonces, está situación se agudiza cuando la política educativa no incluye explícitamente una reflexión tanto ontológica como epistemológica de las personas que dinamizan el hecho educativo. Y esto se observa en la Política de Educación Inclusiva (Ministerio de Educación, 2010b), 
pues si bien reconoce la importancia de tener unos acuerdos mínimos sobre los valores que deben estar presentes en la sociedad y que la escuela debe promover la inclusión, no profundiza en la persona que se pretende incluir, cómo se reconocerá y valorará su diferencia en la educación.

Ahora bien, hay distintos modos de producción de ausencia o no existencia, y es necesario identificarlos, pues estos surgen de la cultura (Santos, 2010). Así, en el contexto salvadoreño, las ausencias pueden darse por diferentes clasificaciones como la raza, el sexo, el lugar de residencia, la condición de discapacidad, entre otros; es decir, se excluye a aquellos que se alejan de la norma establecida por el grupo social dominante. A continuación, se detallan, de acuerdo a la realidad del país.

- Raza. El total de la población indígena en el país es de 13,310 personas provenientes de los pueblos nahúat-pipiles, lencas, kakawira y otros; quienes representan el 0.002\% de la población total (Ministerio de Economía, 2008).

- Sexo. La tasa de asistencia escolar a nivel nacional es de $29.6 \%$ para hombres y $25.6 \%$ en las mujeres, y en el rango de edad de 16 a 18 años se observa mayor diferencia en la inasistencia, 36.4\% para hombres y 39.2\% para mujeres. Luego, en el grupo de 19 años se da el mayor porcentaje de inasistencia, 93.9\% hombres y 94.8\% mujeres (Ministerio de Economía, 2018). Y entre las causas más frecuentes en ambos sexos están no les interesa ir a la escuela o es muy caro, seguidas por trabajar en el caso de los hombres y trabajo doméstico y causas del hogar, en las mujeres.

- Lugar de residencia. La población salvadoreña se distribuye entre la zona urbana, 3,598,836 personas, y 2,145,277 personas en la zona rural, lo que equivaldría a un $63 \%$ y un $27 \%$ respectivamente (Ministerio de Economía, 2008). Mientras que la asistencia educativa en el área urbana a nivel nacional es del 28.4\%, y en el área rural es de 26.0\% (Ministerio de Economía, 2018).

- Situación de discapacidad. En el país existe un total a nivel nacional de 235,302 personas que presentan algún tipo de limitación, ya sea para moverse, escuchar, ver, hablar o desenvolverse de manera autónoma en la vida cotidiana (Ministerio de Economía, 2018).

Entonces, a partir de estas clasificaciones se puede apreciar lo arriesgado que puede ser una política que no visibilice estos elementos, pues se puede continuar con el error de ocultar prejuicios o representaciones sociales que consideran que la diferencia de raza o sexo es una inferioridad insuperable, que el ignorante es quien tiene un limitado conocimiento formal o que si la persona no produce para el sistema no existe (Santos, 2010). Y está construcción sociocultural de desvalorizar a las personas continuará generando exclusión en el día a día, aunque en el discurso se hablé de inclusión, como se ha presentado anteriormente por medio de las consideraciones de análisis propuestas por Terigi (2014).

Finalmente, la inclusión y la equidad se complementan y como propone López (2005), "no hay equidad sin igualdad... la noción de equidad renuncia a la idea de que todos somos iguales, y es precisamente a partir de ese reconocimiento de las diferencias que propone una estrategia para lograr esa igualdad fundamental” (López, 2005, p. 68). Por tanto, en el país no sólo se debe garantizar la igualdad de oportunidades para acceder y permanecer en el sistema educativo, sino también brindar a cada estudiante lo que necesita para 
participar en su aprendizaje. Así, la equidad adquiere un carácter eminentemente político e invita al Estado, a la sociedad civil y demás organizaciones a trabajar para garantizar que la equidad educativa se consolide de manera efectiva por medio de la inclusión, y así poder reconstruir el tejido social.

\section{Referencias}

Aguerrondo, I. (2008). Revisar el modelo: Un desafío para lograr la inclusión. Perspectivas, 38(1), 61-80.

Ainscow, M. y Booth, T. (2000). Índice de inclusión. Desarrollando el aprendizaje y la participación en las escuelas. Bristol: UNESCO.

Ainscow, M., Booth, T. y Dyson, A. (2006). Improving schools, developing inclusion. Abingdon: Routledge.

Asamblea Legislativa de El Salvador. (1983). Constitución de la república. San Salvador: Asamblea Legislativa de El Salvador.

Asamblea Legislativa de El Salvador. (1996). Ley general de educación. San Salvador: Asamblea Legislativa de El Salvador.

Asamblea Legislativa de El Salvador. (2014). Politica nacional de atención integral a las personas con discapacidad. San Salvador: MINED.

Bauman, Z. (2008). Los retos de la educación en la modernidad líquida. Barcelona: Gedisa.

Blanco, R. (2006). La equidad y la inclusión social: Uno de los desafíos de la educación y la escuela hoy. REICE. Revista Iberoamericana sobre Calidad, Eficacia y Cambio en Educación, 4(3), 1-45.

Blanco, R. y Duk, C. (2011). Educación inclusiva en América Latina y el Caribe. Aula, 17(2), 37-55.

Consejo Nacional de Educación. (2016). Plan El Salvador educado. Por el derecho a una educación de calidad. San Salvador: CONED.

Fondo de las Naciones Unidas para la Infancia. (2006). Convención sobre los derechos del niño. Madrid: UNICEF.

Fondo de las Naciones Unidas para la Infancia. (2012). Primera infancia en El Salvador. Estado actual $y$ perspectivas. San Salvador: UNICEF.

Hurtado, K. y Muñoz, C. (2017). El reconocimiento del otro y la gestión escolar. Varela, 17(47), $140-154$.

Krichesky, M. (2006). Escuela y comunidad: Desafios para la inclusión educativa. Buenos Aires: Ministerio de Educación, Ciencia y Tecnología de la Nación.

López, N. (2005). Equidad educativa y desigualdad social. Desafios de la educación en el nuevo escenario latinoamericano. Buenos Aires: IIPE-UNESCO.

López, N. (2007). Escuela y contexto social en América Latina. Cuando la globalización llega al aula. Caracas: Federación Internacional de Fe y Alegría.

López, N. (2009). De relaciones, actores y territorios. Buenos Aires: IIPE-UNESCO.

Miles, S. y Singal, N. (2010). The education for all and icnlusive education debate: Conflict, contradiction or opportunity? International Journal of Inclusive Education, 14(1), 1-15. https://doi.org/10.1080/13603110802265125

Ministerio de Economía. (2008). Censo de población y vivienda 2007. San Salvador: DIGESTYC. 
Ministerio de Economía. (2018). Encuesta de hogares de propósitos múltiples 2017. San Salvador: DIGESTYC.

Ministerio de Educación. (1995). Lineamientos generales del plan decenal 1995-2005. San Salvador: MINED.

Ministerio de Educación. (2000). Desafíos de la educación en el nuevo milenio, reforma educativa en marcha (2000-2005). San Salvador: MINED.

Ministerio de Educación. (2005). Plan nacional de educación 2021. Metas y desafíos para construir el país que queremos. San Salvador: MINED.

Ministerio de Educación. (2008a). Gestión escolar efectiva. San Salvador: MINED.

Ministerio de Educación. (2008b). Normativa de funcionamiento. San Salvador: MINED.

Ministerio de Educación. (2009). Plan social educativo 2009-2014: Vamos a la escuela. San Salvador: MINED.

Ministerio de Educación. (2010a). Adecuación del índice de inclusión al contexto educativo salvadoreño. San Salvador: MINED.

Ministerio de Educación. (2010b). Política de educación inclusiva. San Salvador: MINED.

Ministerio de Educación. (2010c). Política nacional de educación y desarrollo integral de la primera infancia. San Salvador: MINED.

Ministerio de Educación. (2012). Política nacional de desarrollo profesional docente. San Salvador: MINED.

Ministerio de Educación. (2015a). Ejes estratégicos del plan nacional de educación en función de la nación: Educar para el desarrollo de capacidades productivas y ciudadanas. San Salvador: MINED.

Ministerio de Educación. (2015b). Observatorio MINED 2015 sobre los centros educativos de El Salvador. San Salvador: MINED.

Ministerio de Educación. (2015c). Revisión nacional 2015 de la educación para todos: El Salvador. San Salvador: MINED.

Ministerio de Educación. (2016). Política de equidad e igualdad de género. Plan de implementación del ministerio de educación. El Salvador. San Salvador: MINED.

Ministerio de Educación. (2016a). Acuerdo ministerial No. 15-1269. Organigrama general 2015-2019. San Salvador: MINED.

Ministerio de Educación. (2016b). Comités departamentales de apoyo a la inclusión. San Salvador: MINED.

Ministerio de Educación. (2016c). Modelo de escuela inclusiva de tiempo pleno. Documento de sistematización. San Salvador: MINED-EDUCAID.

Ministerio de Educación. (2017a). Centro de orientación y recursos desde el enfoque de inclusión. San Salvador: MINED.

Ministerio de Educación. (2017b). Estrategia educativa docente de apoyo a la inclusión. San Salvador: MINED.

Ministerio de Gobernación. (2013). Política nacional de juventud 2010/2024. San Salvador: INJUVE.

ONU. (2007). Convención sobre los derechos de las personas con discapacidad. Nueva York, NY: ONU.

Opertti, R. (2009, julio). La educación inclusiva, perspectiva internacional y retos de futuro. Comunicación presentada en el Coloquio de historia de la educación la educación especial y social del siglo XIX. Universidad de Navarra. 
Payá Rico, A. (2010). Políticas de educación inclusiva en América Latina. Propuestas, realidades y retos de futuro. Educación Inclusiva, 3(2), 125-142.

Pedro, F. y Puig, I. (1999). Las reformas educativas: Una perspectiva política y comparada. Barcelona: Paidós.

PNUD. (2018). Índice sobre el desarrollo humano El Salvador 2018. ¡Soy joven! ¿̨ ahora qué? San Salvador: PNUD.

Powell, S. y Tutt, R. (2002). When inclusion becomes exclusion. Education 3-13, 30(2), 43-46.

Rambla, X., Ferrer, F., Tarabini, A. y Verger, A. (2008). La educación inclusiva frente a las desigualdades sociales: Un estado de la cuestión y algunas reflexiones geográficas. Perspectivas, 38(1), 81-96.

Sánchez de Horcajo, J. J. (1991). Escuela, sistema y sociedad. Invitación a la sociología de la educación. Madrid: Libertarias/Prodhufi.

Santos, B. D. S. (2010). Descolonizar el saber, reinventar el poder. Montevideo: Trilce.

Telló, C. (2016). Los objetos de estudio de la política educativa. Hacia una caracterización del campo teórico. Buenos Aires: EPUB.

Terigi, F. (2014). La inclusión como problema de las políticas educativas. En M. Feijoó y M. Poggi (Eds.), Educación y políticas sociales. Sinergias para la inclusión (pp. 217-234). Buenos Aires: IIPE-UNESCO.

UNESCO. (2015). La educación para todos, 2000-2015. Logros y desafíos. París: UNESCO.

UNESCO. (2017). Educación para los objetivos de desarrollo sostenible. Objetivos de aprendizaje. París: UNESCO.

Zemelman, H. (2005). Voluntad de conocer. El sujeto y su pensamiento en el paradigma crítico. Barcelona: Anthropos.

\section{Breve CV de la autora}

\section{Cristina Aracely Muñoz Morán}

Maestra en Política y Evaluación Educativa (Universidad Centroamericana José Simeón Cañas), Licenciada en Psicología (Universidad de Deusto), y Profesora en Educación Especial para el nivel básico (Universidad Católica de Occidente), es Catedrática de la Universidad Evangélica de El Salvador en el Departamento de Educación Especial. Su labor de investigación está relacionada con el desarrollo de la educación inclusiva a través de las políticas educativas, las prácticas pedagógicas, la participación de actores sociales y la gestión escolar, teniendo como punto de referencia el reconocimiento de la persona en el contexto educativo. ORCID ID: https://orcid.org/OOOO-OOO2-1785-4884. Email: cristina.munoz@uees.edu.sv 